\title{
Optimizing Antibiotic Prescribing for Acute Respiratory Tract Infection in German Primary Care: Study Protocol for Evaluation of the RESIST Program
}

Christin Löffler ${ }^{1}, \mathrm{PhD}$; Antje Krüger ${ }^{1}, \mathrm{MA}$; Anne Daubmann ${ }^{2}$, MSc; Julia Iwen ${ }^{3}, \mathrm{PhD}$; Marc Biedermann ${ }^{4}, \mathrm{MSc}$; Maike Schulz ${ }^{5}$, MSc; Karl Wegscheider ${ }^{2}$, PhD, Prof Dr; Attila Altiner ${ }^{1}$, MD, Prof Dr; Gregor Feldmeier ${ }^{1}$, MD, PhD; Anja Wollny ${ }^{1}, \mathrm{PhD}$

\footnotetext{
${ }_{1}^{1}$ Institute of General Practice, Rostock University Medical Center, Rostock, Germany

${ }^{2}$ Institute of Medical Biometry and Epidemiology, University Medical Center Hamburg- Eppendorf, Hamburg, Germany

${ }^{3}$ Association of Substitute Health Funds (Vdek), Berlin, Germany

${ }^{4}$ National Association of Statutory Health Insurance Physicians, Berlin, Germany

${ }^{5}$ Central Research Institute of Ambulatory Health Care in Germany, Berlin, Germany
}

\section{Corresponding Author:}

Christin Löffler, $\mathrm{PhD}$

Institute of General Practice

Rostock University Medical Center

Doberaner Str 142 (Villa im Hof)

Rostock, 18057

Germany

Phone: 49381494 ext 2481

Email: christin.loeffler@med.uni-rostock.de

\section{Abstract}

Background: The emergence and increased spread of microbial resistance is a major challenge to all health care systems worldwide. In primary care, acute respiratory tract infection (ARTI) is the health condition most strongly related to antibiotic overuse.

Objective: The RESIST program aims at optimizing antibiotic prescribing for ARTI in German primary care. By completing a problem-orientated online training course, physicians are motivated and empowered to utilize patient-centered doctor-patient communication strategies, including shared decision making, in the treatment of patients with ARTI.

Methods: RESIST will be evaluated in the form of a nonrandomized controlled trial. Approximately 3000 physicians of 8 (out of 16) German federal states can participate in the program. Patient and physician data are retrieved from routine health care data. Physicians not participating in the program serve as controls, either among the 8 participating regional Associations of Statutory Health Insurance Physicians (control group 1) or among the remaining associations not participating in RESIST (control group 2). Antibiotic prescription rates before the intervention (T0: 2016, 1st and 2nd quarters of 2017) and after the intervention (T1: 3rd quarter of 2017 until 1st quarter of 2019) will be compared. The primary outcome measure is the overall antibiotic prescription rate for all patients insured with German statutory health insurance before and after provision of the online course. The secondary outcome is the antibiotic prescription rate for coded ARTI before and after the intervention.

Results: RESIST is publicly funded by the Innovations funds of the Federal Joint Committee in Germany and was approved in December 2016. Recruitment of physicians is now completed, and a total of 2460 physicians participated in the intervention. Data analysis started in February 2020.

Conclusions: With approximately 3000 physicians participating in the program, RESIST is among the largest real-world interventions aiming at reducing inadequate antibiotic prescribing for ARTI in primary care. Long-term follow up of up to 21 months will allow for investigating the sustainability of the intervention.

Trial Registration: ISRCTN Registry ISRCTN13934505; http://www.isrctn.com/ISRCTN13934505

International Registered Report Identifier (IRRID): RR1-10.2196/18648 
(JMIR Res Protoc 2020;9(9):e18648) doi: 10.2196/18648

\section{KEYWORDS}

antibacterial agents; respiratory tract infection; upper respiratory tract infection; lower respiratory tract infection; primary care; primary health care; physician-patient relation; shared decision making; antibiotic resistance

\section{Introduction}

\section{Background}

Growing microbial resistance is a major challenge of all health care systems worldwide. The overuse and misuse of antibiotics in human and veterinary medicine accelerates this development $[1,2]$. In primary care, acute respiratory tract infection (ARTI) is the health condition most strongly related to antibiotic overuse $[3,4]$. This is a striking fact, as most cases of ARTI are caused by viruses and/or are self-limiting [5-7]. Considerable geographical variations in antibiotic prescribing for ARTI exist. In Europe, prescribing rates for ARTI range from below $40 \%$ in the Netherlands, Scandinavia, and Germany to up to $60 \%$ in the United Kingdom and Ireland, and reach up to almost $80 \%$ in southern Europe [8]. Reasons for inadequate prescribing include the physician's overestimation of patient expectations toward antibiotic treatment as well as a deceptive "safety culture" in certain cases $[9,10]$.

A considerable number of interventions to reduce inadequate prescribing for ARTI have been developed and evaluated. Overall, the main intervention strategies applied to date include laboratory and point of care testing (POCT), provider or patient education, computerized clinical decision support, prescribing feedback, delayed prescribing, and communication training. There is now sufficient evidence for the effectiveness of communication skills training and laboratory testing/POCT to reduce antibiotic prescribing for ARTI $[11,12]$. In addition, there is evidence that the effect of these interventions differs according to the level of antibiotic prescribing for ARTI in a given setting. High-prescribing countries benefit from laboratory testing/POCT, whereas medium- and low-prescribing countries show higher effects for communication training [12].

In parallel to the design and evaluation of interventional approaches, an increasing number of nations and supranational bodies have started initiating programs of antimicrobial stewardship. These usually include public information campaigns, education for health care providers, local surveillance of antibiotic resistance, and the provision of research grants to generate evidence necessary to improve antimicrobial stewardship [13]. Along with these initiatives, the German DART 2020 strategy was developed. Apart from strengthening a One Health approach to antimicrobial stewardship by addressing both human and veterinary medicine, supporting research is a major aim of the initiative. In this context, since 2016, the German Innovationsfonds granted several programs and trials addressing antimicrobial stewardship. The RESIST program is one of these programs. Within the program, approximately 3000 physicians of 8 (out of 16) German federal states can participate in online training aiming at patient-centered medical care for patients suffering from ARTI. Nationally and internationally, RESIST is one of the largest scientifically evaluated health intervention programs conducted to date. This protocol describes how the program is being evaluated.

\section{Objectives}

RESIST aims at optimizing antibiotic prescribing for ARTI in German primary care. By completing a problem-orientated online training course, physicians are motivated and empowered to utilize patient-centered doctor-patient communication strategies, including shared decision making, in the treatment of patients with ARTI. Additionally, the courses provide current data on microbial resistance development and on rational antibiotic use. Moreover, physicians receive information materials for their patients as well as regional prescribing feedback. Based on this protocol, we are assessing whether the RESIST program reduces the number of antibiotics prescribed and, in the case of antibiotic use, impacts the choice of substance. The primary outcome measure is the antibiotic prescription rate for all patients insured with German statutory health insurance before and after provision of the online course. The secondary outcome is the antibiotic prescription rate for coded ARTI before and after the intervention. In addition, using individual qualitative interviews and focus group discussions, the perspective and perceptions of patients, physicians, and practice staff on the program will be evaluated.

\section{Methods}

\section{Study Design}

As RESIST is primarily a real-world project aiming to address as many participants as funding allows, it is evaluated in the form of a nonrandomized controlled trial. Patient and physician data are retrieved from routine health care data used by the Central Research Institute of Ambulatory Health Care in Germany ( $\mathrm{Zi})$. The $\mathrm{Zi}$ continuously analyzes prescription and diagnoses data on behalf of all German regional Associations of Statutory Health Insurance Physicians. The data analyzed include information on diagnosis as coded in practices and prescriptions as collected in pharmacies. Physicians not participating in the program serve as controls, either among the 8 statewide participating Associations of Statutory Health Insurance Physicians (control group 1) or among the federal states not eligible for participation in RESIST (control group 2). Antibiotic prescription rates before the intervention (T0: $2016,1^{\text {st }}$ and $2^{\text {nd }}$ quarters of 2017) and after the intervention (T1: $3^{\text {rd }}$ quarter of 2017 until $1^{\text {st }}$ quarter of 2019) are compared.

\section{Recruitment}

Recruitment of participating physicians is undertaken in 8 German federal states and regions (Bavaria, Baden-Wuerttemberg, Lower Saxony, North Rhine, Westphalia-Lippe, Brandenburg, Mecklenburg-Western Pomerania, and Saarland), assisted by the respective regional Association of Statutory Health Insurance Physicians. General 
practitioners, primary care pediatricians, and practice-based otorhinolaryngologists can enroll in the RESIST program on a voluntary "first-come, first-served" basis. The maximum number of participating physicians per region is related to the size of the regional association. In total, we aim to include up to 3000 physicians in the program.

\section{Participants}

Physicians successfully completing the RESIST online training course are compared to nonparticipating physicians.

\section{Blinding}

Participating physicians are not blinded as they enroll voluntarily in the RESIST program.

\section{Intervention}

The intervention is based on an online training course consisting of three modules. Each module can be completed within approximately 1 hour. Module 1 focuses on patient-centered doctor-patient communication, and points to typical communicative situations when treating patients with ARTI. Within the module, physicians are encouraged to reflect upon these situations and to question perceived pressure to prescribe antibiotics. Modules 2 and 3 deal with rational antibiotic use in the case of upper and lower respiratory tract infections. After passing the Continued Medical Education-accredited course, physicians receive posters and brochures on rational antibiotic use to display them in their practices or to use them during consultations. Physicians successfully completing the course receive a one-time allowance. In addition, physicians receive further reimbursement for up to 20 patients with ARTI per quarter, which they document as treated according to the strategies adopted after participating in the online training. In addition, participating physicians receive a feedback report on aggregated data on regional antibiotic prescribing patterns. Figures are compared between intervention and control groups. See Multimedia Appendix 1 for more details on each component and module of the intervention [14].

\section{Control Group}

Physicians not participating in the program serve as controls, either among the 8 participating regional Associations of Statutory Health Insurance Physicians (control group 1) or among the remaining associations not participating in RESIST (control group 2).

\section{Outcomes}

The primary outcome measure is the overall antibiotic prescription rate of all patients insured with a German statutory health insurance before and after the provision of online courses. Secondary outcomes are (a) the antibiotic prescription rate for ARTI in patients aged $\geq 1$ year before and after the intervention; (b) the antibiotic prescription rate based on different types of ARTI (eg, otitis media, bronchitis, pneumonia; see Table 1) before and after the intervention in patients aged $\geq 1$ year; and (c) quality of antibiotic prescribing for ARTI measured by internationally accredited quality indicators of the European Surveillance of Antimicrobial Consumption Project (ESAC) [15], again before and after the intervention. 
Table 1. International Classification of Diseases codes relevant for diagnosing acute respiratory tract infection.

\begin{tabular}{|c|c|}
\hline Code & Diagnosis \\
\hline J00 & Acute nasopharyngitis (common cold) \\
\hline J01 & Acute sinusitis \\
\hline $\mathrm{J} 02$ & Acute pharyngitis \\
\hline J03 & Acute tonsillitis \\
\hline J04 & Acute laryngitis and tracheitis \\
\hline J06 & Acute upper respiratory infections of multiple and unspecified site \\
\hline J09 & Influenza due to identified zoonotic or pandemic influenza virus \\
\hline $\mathrm{J} 10$ & Influenza due to identified seasonal influenza virus \\
\hline $\mathrm{J} 11$ & Influenza, virus not identified \\
\hline $\mathrm{J} 12$ & Viral pneumonia, not elsewhere classified \\
\hline $\mathrm{J} 13$ & Pneumonia due to Streptococcus pneumoniae \\
\hline $\mathrm{J} 14$ & Pneumonia due to Haemophilus influenzae \\
\hline $\mathrm{J} 15$ & Bacterial pneumonia, not elsewhere classified \\
\hline $\mathrm{J} 16$ & Pneumonia due to other infectious organisms, not elsewhere classified \\
\hline $\mathrm{J} 17$ & Pneumonia in diseases classified elsewhere \\
\hline $\mathrm{J} 18$ & Pneumonia, organism unspecified \\
\hline $\mathrm{J} 20$ & Acute bronchitis \\
\hline $\mathrm{J} 21$ & Acute bronchiolitis \\
\hline $\mathrm{J} 22$ & Unspecified acute lower respiratory infection \\
\hline $\mathrm{J} 40$ & Bronchitis, not specified as acute or chronic \\
\hline H65 & Nonsuppurative otitis media \\
\hline H66 & Suppurative and unspecified otitis media \\
\hline
\end{tabular}

\section{Sample Size}

With respect to the primary outcome, a reduction of the physicians' overall antibiotic prescription rate by $1 \%$ is assumed for RESIST (from $33.8 \%$ in the control group 1 to $32.8 \%$ in the intervention group). Without consideration of cluster effects, 2 $\times 34,865$ patients are needed to show this effect with a power of $80 \%$ and a type I error of 5\% (two-sided). Assuming an intracluster correlation (ICC) of $8 \%$ [16] and an average cluster size of 160 patients per practice, a design effect of 13.72 is determined. Overall, the sample size increases to $2 \times 478,400$ patients $(956,800$ patients in total). Thus, $2 \times 2990$ practices (5980 in total) are needed for the evaluation.

For the secondary outcome, a reduction of 3\% in the physicians' antibiotic prescription rate for ARTI is expected (from 30.5\% in the control group 1 to $27.5 \%$ in the intervention group). Assuming a power of $80 \%$ and a type I error of 5\% (two-sided), $2 \times 3590$ patients are required. With an ICC of $8 \%$ [16] and an average cluster size of 127 patients (diagnosed with ARTI) per practice, the sample size increases to $2 \times 39,751$ patients $(79,502$ patients in total). Hence, $2 \times 313$ practices are needed $(626$ practices in total). The sample size necessary to investigate the effect of the intervention on the primary outcome will be adequate to investigate its effect on the secondary outcome as well.

\section{Data Collection, Completeness, and Quality}

The trial is based on pseudonymized routine data provided by the $\mathrm{Zi}$. The dataset is based on aggregated practice data. Information collected includes: age groups and sex of patients, ARTI diagnoses (grouped and individual diagnoses), antibiotic prescriptions, group affiliation of the physician (intervention vs control), region, level of urbanization, and type of medical insurance of the patient.

\section{Statistical Methods}

Data analyses will be performed in accordance with the STROBE statement [17]. For descriptive statistics, we will report the primary and the secondary outcome for the whole sample and by treatment allocation. Sampling units are practices. This evaluation is designed to confirm the two-sided primary hypothesis that the physicians' antibiotic prescription rate after $\mathrm{T} 1$, regardless of the diagnosis, is lower in the intervention group than in control group 1. For investigating the primary outcome, we will use a negative binomial regression with the absolute number of annual antibiotic prescriptions as the dependent variable, the number of patients as the offset variable, and the antibiotic prescription rate before the introduction of RESIST as a covariate. To avoid bias, we will include region, specialization of the physician, proportion of male patients, proportion of different age classes ( $\leq 7$ years, 8 to 18 years, 19 to 65 years, $>66$ years), proportion of insurance status (member, 
pensioner, noncontributory dependents), and the interaction between the proportion of male patients and region as additional covariates. The comparison between the intervention group and control group 1 is considered in a confirmatory manner. The comparison between the intervention group and control group 2 is additionally examined as a sensitivity analysis. The model for the secondary outcomes will be formulated in the same manner. The results will be represented as incident rate ratios with their $95 \%$ CIs and $P$ values. Statistical analyses will be carried out with SAS, version 9.4 or the newer version (SAS Institute, Cary, NC, USA).

With respect to quality indicators (according to ESAC), sampling units are practices. The analysis will be carried out in the same way as the analysis of the primary and secondary outcomes considering the requirements of each indicator.

\section{Process Evaluation}

Qualitative interviews and focus group discussions will be used to investigate the perspective and perceptions of patients, physicians, and practice staff on the program. First and foremost, the qualitative evaluation focuses on factors fostering or hindering the implementation of the program. To this end, one to two focus group discussions per participating federal state or region will be conducted. Focus group discussions will include 6 to 8 physicians or practice staff members and are assumed to last about 60 to 90 minutes each.

To explore the patients' perspectives, we will additionally conduct up to 50 narrative interviews by telephone. We will interview patients treated for ARTI by physicians participating in the RESIST program. We assume that phone calls will last about 10 to 30 minutes each.

Both focus group discussions and qualitative interviews will be recorded, transcribed verbatim, and analyzed based on content analysis [18].

To verify the findings of this qualitative process evaluation at large, a questionnaire for physicians and for patients suffering from ARTI will be developed. We aim to survey about 1000 individuals in each group. Physicians will be approached directly and will be rewarded monetarily for their support. Patient questionnaires will be distributed via participating physicians.

\section{Harms}

The careful and sustainable prescription of antibiotics for ARTI is based on national and international clinical guidelines. Beyond that, the decision to prescribe antibiotics in the individual case remains the choice of the physician. Thus, harms are not expected.

\section{Study Registration}

The program was retrospectively registered on December 6, 2018 at the ISRCTN Registry under reference ISRCTN13934505.

\section{Ethical Approval and Consent to Participate}

The evaluation was approved by the ethics committee at Rostock University Medical Center in June 2017 (Reference: A
2017-0090). Participating physicians provide written informed consent. However, no informed consent is necessary from the patients, as pseudonymized and de facto anonymized routine data are used based on $§ 80$ SGB X.

\section{Availability of Data and Materials}

The datasets generated and analyzed during the current study will be available upon request from the Zi (email: zi@zi.de) in the form of aggregated anonymized raw data collected from March 2020 to March 2025. Data will be shared upon request for the purpose of academic research and scientific analyses (such as meta-analysis).

\section{Results}

RESIST is publicly funded by the Innovations funds of the Federal Joint Committee (G-BA) in Germany (funding code: 01NVF16005) and was approved in December 2016.

Recruitment of physicians is completed. A total of 2460 physicians participated in the intervention. Data analysis started in February 2020.

\section{Discussion}

With up to 3000 physicians participating in the program, RESIST is among the largest real-world interventions aiming at reducing inadequate antibiotic prescribing for ARTI in primary care. Long-term follow up of up to 21 months will allow for investigating the sustainability of the intervention.

As RESIST utilizes an open-label nonrandomized evaluation concept, potential bias has to be carefully addressed. Bias may arise from the fact that physicians most interested in reducing inadequate antibiotic prescriptions are also likely to be those most motivated to take part in RESIST. To counteract this, we make use of an attractive incentivization and reimbursement scheme. In addition, the inclusion of comparable practices not participating in the program (both among participating regions and among those not participating in RESIST) allows for control of this potential source of bias.

We will also minimize bias due to changed coding. For example, a clinically identical patient treated with antibiotics might be coded with lower respiratory tract infection initially but with pneumonia after the intervention, leading to an overestimation of the interventional effect. By measuring the overall antibiotic prescribing rate of antibiotics without regard to any specific disease as the primary outcome measure, we can exclude bias due to coding phenomena.

Last but not least, the RESIST program joins the ranks of other current initiatives to reduce inadequate antibiotic prescribing. Thus, contamination effects between the projects are possible. However, by including a high number of physicians and practices among half of all German states, we believe to be able to address this potential source of bias adequately. 


\section{Acknowledgments}

We thank all physicians participating in the trial. RESIST is publicly funded by the Innovations funds of the Federal Joint Committee (G-BA) in Germany (funding code: 01NVF16005). The sponsor had no influence on the design of the study or the writing of the manuscript. The funder also does not have any influence in the collection, analysis, or interpretation of the data.

\section{Authors' Contributions}

AA and JI are responsible for the design of the study. CL and AK wrote the manuscript. All authors critically read and approved the final manuscript.

\section{Conflicts of Interest}

None declared.

\section{Multimedia Appendix 1}

Components $1-5$ of the intervention.

[DOCX File, 23 KB-Multimedia Appendix 1]

\section{References}

1. Schulz M, Kern W, Hering R, Schulz M, Bätzing-Feigenbaum J. Antibiotikaverordnungen in der ambulanten Versorgung in Deutschland bei bestimmten Infektionserkrankungen in 2009 - Tiel 1 und 2. Versorgungsatlas. Berlin: Zentralinstitut für die kassenärztliche Versorgung in Deutschland (Zi); 2009. URL: https://www.versorgungsatlas.de/themen/ alle-analysen-nach-datum-sortiert/?tab=6\&uid=46

2. Bundesministerium für Gesundheit, Bundesministerium für Ernährung und Landwirtschaft, Bundesministerium für Bildung und Forschung. DART 2020 - Antibiotika-Resistenzen bekämpfen zum Wohl von Mensch und Tier. Strategiepapier. Berlin; 2015. URL: https://www.bundesgesundheitsministerium.de/themen/praevention/antibiotika-resistenzen/ antibiotika-resistenzstrategie.html

3. Bundesamt für Verbraucherschutz und Lebensmittelsicherheit. GERMAP 2012 - Antibiotika-Resistenz und -Verbrauch. In: Forschungsbericht. Rheinbach: Antiinfectives Intelligence; 2014.

4. Schwabe U, Paffrath D. Arzneiverordnungs-Report 2015. Berlin, Heidelberg: Springer-Verlag; 2015.

5. Wirkstoff Aktuell - Rationale Antibiotikatherapie bei Infektionen der oberen Atemwege. Kassenärztliche Bundesvereinigung. 2012. URL: https://www.kbv.de/media/sp/Antibiotika Infektionen obere Atemwege.pdf [accessed 2019-12-05]

6. Wirkstoff Aktuell - Rationale Antibiotikatherapie bei Infektionen der unteren Atemwege. Kassenärztliche Bundesvereinigung. 2017. URL: http://www.akdae.de/Arzneimitteltherapie/WA/Archiv/Antibiotika-LRTI.pdf [accessed 2019-12-05]

7. Altiner A, Bell J, Duerden M, Essack S, Kozlov R, Noonan L, et al. More action, less resistance: report of the 2014 summit of the Global Respiratory Infection Partnership. Int J Pharm Pract 2015 Feb 25;23(5):370-377. [doi: 10.1111/ijpp.12177]

8. Coenen S, Ferech M, Haaijer-Ruskamp FM, Butler CC, Vander Stichele RH, Verheij TJM, et al. European Surveillance of Antimicrobial Consumption (ESAC): quality indicators for outpatient antibiotic use in Europe. Qual Safe Health Care 2007 Dec 01;16(6):440-445. [doi: 10.1136/qshc.2006.021121] [Medline: 18055888]

9. Altiner A. Acute cough: a qualitative analysis of how GPs manage the consultation when patients explicitly or implicitly expect antibiotic prescriptions. Family Pract 2004 Oct 01;21(5):500-506. [doi: 10.1093/fampra/cmh505] [Medline: 15367471]

10. Altiner A, Brockmann S, Sielk M, Wilm S, Wegscheider K, Abholz H. Reducing antibiotic prescriptions for acute cough by motivating GPs to change their attitudes to communication and empowering patients: a cluster-randomized intervention study. J Antimicrob Chemother 2007;60(3):638-644. [doi: 10.1093/jac/dkm254] [Medline: 17626023]

11. Drekonja DM, Filice GA, Greer N, Olson A, MacDonald R, Rutks I, et al. Antimicrobial Stewardship in Outpatient Settings: A Systematic Review. Infect Control Hosp Epidemiol 2014 Dec 22;36(2):142-152. [doi: 10.1017/ice.2014.41] [Medline: 25632996]

12. Köchling A, Löffler C, Reinsch S, Hornung A, Böhmer F, Altiner A, et al. Reduction of antibiotic prescriptions for acute respiratory tract infections in primary care: a systematic review. Implementation Sci 2018 Mar 20;13(1):47. [doi: 10.1186/s13012-018-0732-y] [Medline: 29554972]

13. European strategic action plan on antibiotic resistance. World Health Organization Regional Committee for Europe. 2011. URL: http://www.euro.who.int/ data/assets/pdf file/0008/147734/wd14E AntibioticResistance 111380.pdf [accessed 2019-12-05]

14. Hoffmann TC, Glasziou PP, Boutron I, Milne R, Perera R, Moher D, et al. Better reporting of interventions: template for intervention description and replication (TIDieR) checklist and guide. BMJ 2014 Mar 07;348:g1687-g1687. [doi: 10.1136/bmj.g1687] [Medline: 24609605]

15. Disease-specific antibiotic prescribing quality indicators report. European Centre for Disease Prevention and Control. 2010. URL: https://ecdc.europa.eu/sites/portal/files/media/en/healthtopics/antimicrobial-resistance-and-consumption/ 
antimicrobial-consumption/publications-documents/Documents/

ESAC-Net-archive-report disease specific antibiotic prescribing quality indicators.pdf [accessed 2019-12-05]

16. Altiner A, Berner R, Diener A, Feldmeier G, Köchling A, Löffler C, et al. Converting habits of antibiotic prescribing for respiratory tract infections in German primary care - the cluster-randomized controlled CHANGE-2 trial. BMC Fam Pract 2012 Dec 20;13(1):124. [doi: 10.1186/1471-2296-13-124] [Medline: 23256712]

17. von Elm E, Altman DG, Egger M, Pocock SJ, Gøtzsche PC, Vandenbroucke JP. The Strengthening the Reporting of Observational Studies in Epidemiology (STROBE) statement: guidelines for reporting observational studies. Lancet 2007 Oct;370(9596):1453-1457. [doi: 10.1016/s0140-6736(07)61602-x]

18. Flick U. Qualitative Sozialforschung: Eine Einführung. Reinbek bei Hamburg: Rowohlt Taschenbuch Verlag; 2016.
Abbreviations
ARTI: acute respiratory tract infection
ESAC: European Surveillance of Antimicrobial Consumption Project
ICC: intracluster correlation
POCT: point of care testing
Zi: Central Research Institute of Ambulatory Health Care in German

Edited by G Eysenbach; submitted 10.03.20; peer-reviewed by AM Pedro, G Tramper-Stranders; comments to author 12.06.20; revised
version received 01.07.20; accepted 14.07.20; published 30.09.20
Please cite as:
Löffler C, Krüger A, Daubmann A, Iwen J, Biedermann M, Schulz M, Wegscheider K, Altiner A, Feldmeier G, Wollny A
Optimizing Antibiotic Prescribing for Acute Respiratory Tract Infection in German Primary Care: Study Protocol for Evaluation of
the RESIST Program
JMIR Res Protoc 2020;9(9):e18648
URL: $\underline{\text { http://www.researchprotocols.org/2020/9/e18648/ }}$
doi: $\underline{10.2196 / 18648}$
PMID: $\underline{32996888}$

(C) Christin Löffler, Antje Krüger, Anne Daubmann, Julia Iwen, Marc Biedermann, Maike Schulz, Karl Wegscheider, Attila Altiner, Gregor Feldmeier, Anja Wollny. Originally published in JMIR Research Protocols (http://www.researchprotocols.org), 30.09 .2020$. This is an open-access article distributed under the terms of the Creative Commons Attribution License (https://creativecommons.org/licenses/by/4.0/), which permits unrestricted use, distribution, and reproduction in any medium, provided the original work, first published in JMIR Research Protocols, is properly cited. The complete bibliographic information, a link to the original publication on http://www.researchprotocols.org, as well as this copyright and license information must be included. 